\title{
An Ensemble of Atomic Fountains
}

\author{
Steven Peil, Scott Crane, James Hanssen, Thomas B. Swanson and Christopher R. Ekstrom \\ Clock Development Division \\ United States Naval Observatory \\ Washington, DC 20392
}

\begin{abstract}
Four rubidium fountains have been in operation for the past 1.2 years at USNO. Individual fountain performance, applications to timekeeping and to tests of Local Position Invariance are presented.
\end{abstract}

\section{INTRODUCTION}

The U. S. Naval Observatory provides precise time to a variety of users by relying on a clock ensemble consisting of approximately 25 hydrogen masers and 80 high performance commercial cesium-beam clocks. A program to develop fountain clocks to add to the ensemble was started a number of years ago. As of this proceeding, four rubidium fountains have been in operation for 1.2 years, during which their performance has been assessed and they have gradually been introduced into the USNO timescale. Additionally, all four fountains are being reported to the BIPM as of January 2012 (December 2011 data and onward). Three more fountains are under construction and will reside at the USNO's Alternate Master Clock facility.

\section{OPERATIONAL OVERVIEW}

The four fountains in operation are designated as NRF2, NRF3, NRF4 and NRF5. These systems were built in two generations, according to slightly different designs, as discussed in previous reports [1]. All four began continuous operation in a dedicated clock facility in March 2011.

Each fountain has a local oscillator that is referenced to a hydrogen maser, which also serves as the reference for an auxiliary output generator (AOG). Every 20 seconds, the average relative frequency of the fountain and maser is used to steer the AOG output, providing a continuous nominal $5 \mathrm{MHz}$ signal that reflects the fountain frequency and is measured against the USNO master clock and other clocks in the ensemble. The fountain switches to holdover mode, in which the maser serves as a flywheel, and back automatically. Detection of some problem in operation will cause the fountain to apply a steer that is the average frequency over the previous hour; resolution of the problem results in the fountain steering normally again.

The combination of this ability to rely on the maser as a flywheel and the general robustness of the fountains over the past 1.2 years has resulted in high uptime. The percentage of time over the past 14 months that each fountain has generated a good, steered output is $98.7 \%, 99.6 \%, 97.6 \%$ and $100 \%$ for
NRF2 through NRF5. Some of the down time was not indicative of failure, but rather corresponded to intentional intervention for upgrades and modifications.

\section{FOUNTAIN PERFORMANCE}

\section{A. Second generation design}

The relative performance of the second generation of operational fountains, NRF4 and NRF5, is characterized in Fig. 1, where we show the relative phase over the past 14 months and the corresponding Allan deviation plot. The average frequency difference between the fountains has been $2 \times 10^{-16}$, demonstrating good agreement in the frequencies of two clocks built according to the same design. The Allan deviation curve reflects white frequency noise at an average level of $1.9 \times 10^{-13} / \sqrt{ } \tau$ for each fountain, reaching the high $10^{-17} \mathrm{~s}$ at 85 days. The data is consistent with no relative drift between the fountains at the level of $1 \times 10^{-18} /$ day.

(a)
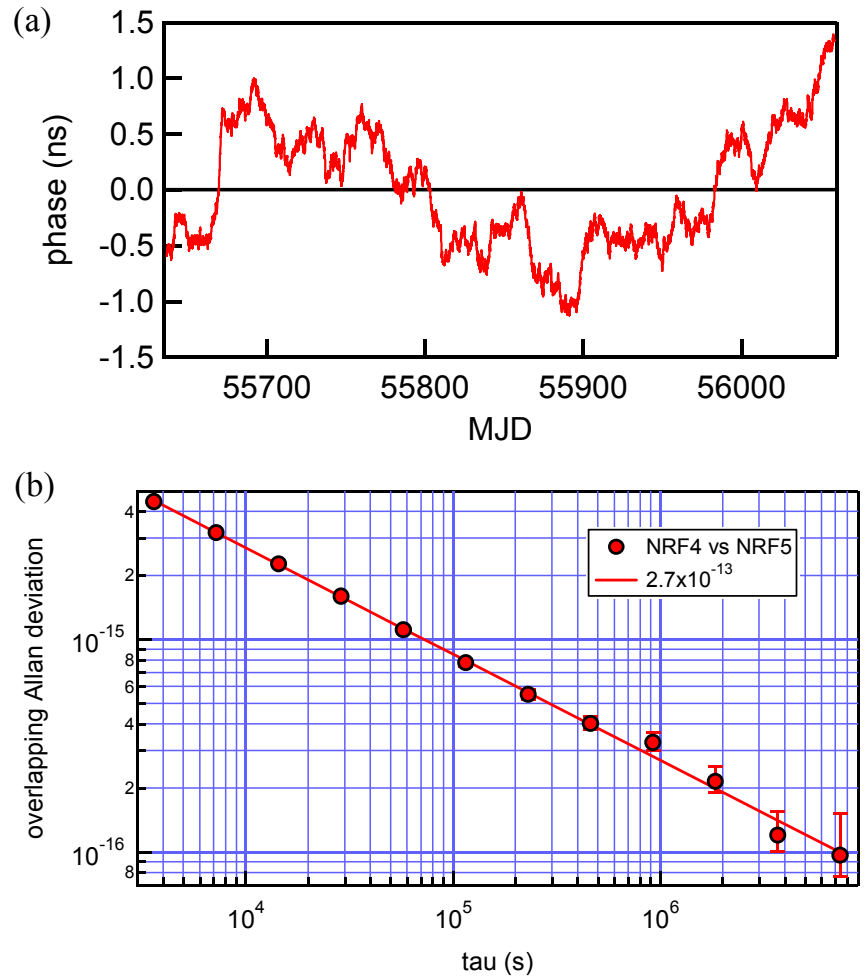

Figure 1. (a) Relative phase of NRF4 and NRF5 over 1.2 years after removing an average frequency difference of $2 \times 10^{-16}$. (b) Plot of overlapping Allan deviation versus averaging time tau. 


\section{B. First generation design}

Several discrete changes in frequency were observed over the past 14 months in each of NRF2 and NRF3, which were built according to an earlier design. The total number of frequency changes was 5 , with size typically ranging from $5 \times 10^{-16}$ to $1.5 \times 10^{-15}$. Unambiguous identification of a fountain exhibiting a change in frequency is possible with our ensemble; by analyzing the performance of a clock compared to the average of the other three, occasional discrete changes in frequency of the type observed are easily identifiable, as illustrated in Fig. 2(a). For the purpose of generating a stable frequency reference, problematic behavior in a fountain can be accounted for (recharacterization of the clock), and the result is four fountains that each reflect the behavior of a stationary fountain mean; see Fig 2(b).

The recharacterization process should not be necessary on a regular basis; the difference in behavior of the two generations of fountain design indicates that the issue of frequency changes can be solved by implementing the design improvements from the second generation in NRF2 and NRF3. Indeed, we have strong indication that additional isolation of RF and microwave signals will eliminate this problem.
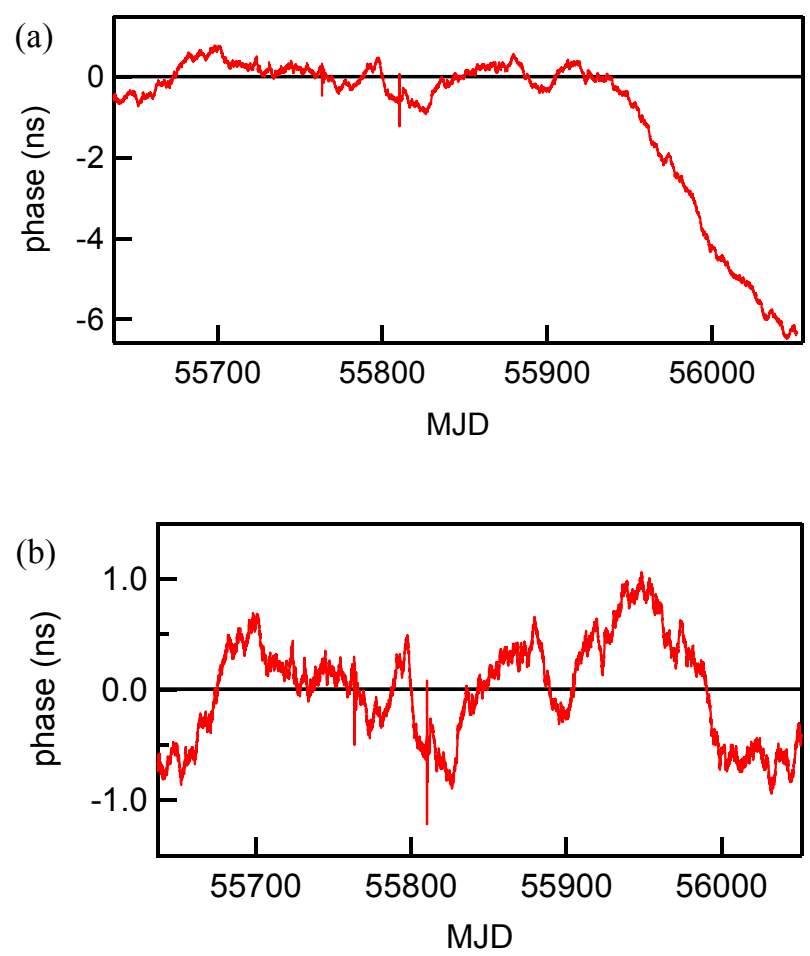

Figure 2. (a) Relative phase of NRF2 and the average of the other three fountains. The change in slope corresponds to a frequency change in NRF2 of $5 \times 10^{-16}$. (b) Relative phase of NRF2 and the average of the other three fountains after recharacterization of NRF2 and NRF3 at a total of five times.

\section{Environmental and other potential frequency shifts}

We routinely record and monitor certain environmental and experimental parameters such as temperature of the clock rooms, number of atoms and magnetic field. It is easy to convert the fluctuations in these three parameters into corresponding fluctuations of the clock frequency in one of our rubidium fountains using a simple model. The resulting frequency instability from the variations in these parameters over the past 14 months is below $1 \times 10^{-17}$, which is well below any level of instability in which we are interested.

\section{FOUNTAIN MEAN AND UTC}

The USNO fountains have been used over the past 14 months to help generate and steer UTC(USNO). In this section we compare the behavior of a rudimentary fountain mean, an unweighted average of the four fountains after recharacterization of NRF2 and NRF3, with UTC. In Fig. 4(a), the relative phase of UTC and the fountain mean is shown for the past 14 months, after removing a relative phase and frequency; we use UTC(USNO) as a transfer oscillator for this calculation. The last six months of this interval is shown in Fig. 4(b), with a finer vertical scale, showing that the residuals between the two timescales are within 1 ns over this period. The fountain mean shows similar behavior versus UTC over the first six months of the total interval, but the relative frequency of the fountain mean and UTC changes by about $2 \times 10^{-15}$ over the entire comparison. This is claimed to be well explained by a change in the algorithm for generating UTC made by the BIPM [2].
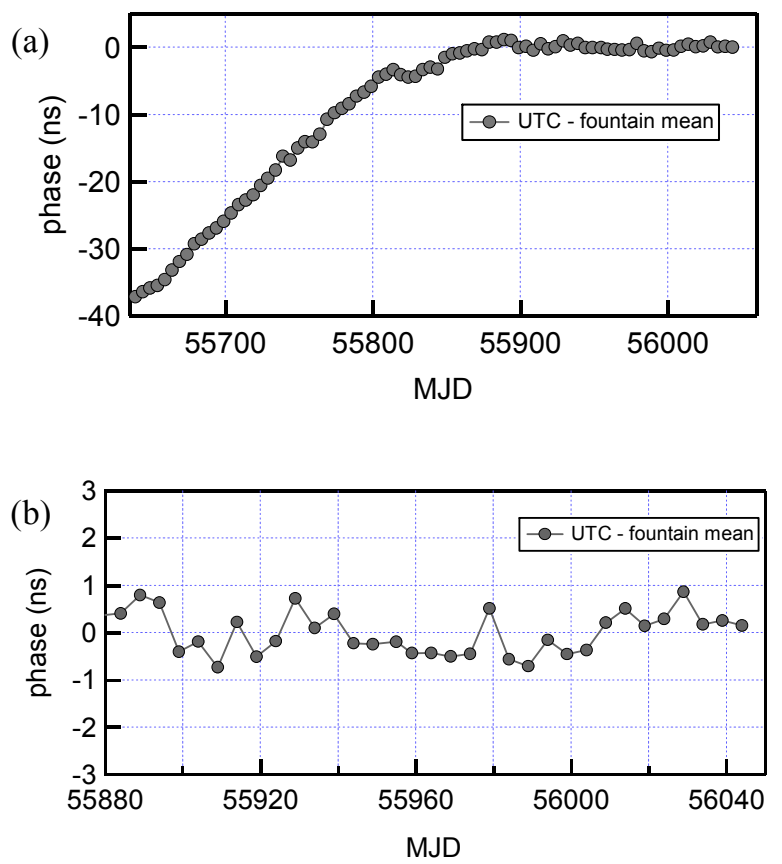

Figure 3. (a) Residual phase of the fountian mean versus UTC over 14 month interval after removing a relative phase and frequency. (b) The last 6 months of the plot in (a). 
We have also compared the stability of the frequency of the fountain mean against the primary standards contributing to TAI. A linear fit to the relative frequencies shows no drift with a limit of about $3 \times 10^{-18} /$ day, as shown in Fig. 4 .

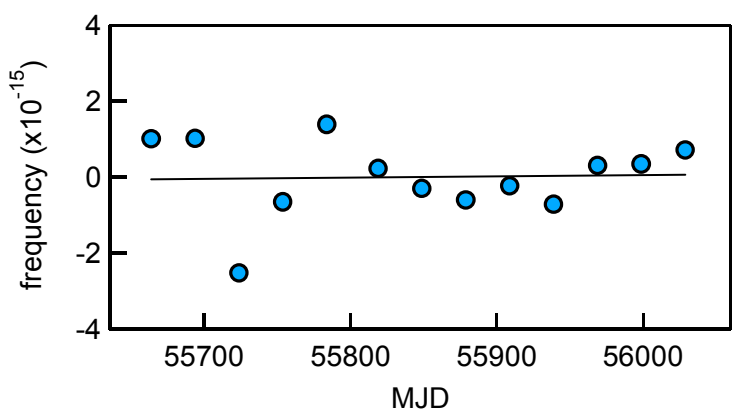

Figure 4. Relative frequency of fountain mean and primary standards that contribute to TAI, along with linear fit.

\section{LOCAL POSITION INVARIANCE}

Atomic clocks have been used to test general relativity and, more generally, the Einstein Equivalence Principle in a variety of ways. One such test is to look for violations of Local Position Invariance (LPI), which states that results of nongravitational measurements are independent of where they are made. Violation of LPI may manifest itself in an anomalous gravitational redshift of the frequency of an atomic clock. While this may be searched for using the dependence of the gravitational redshift of a clock versus gravitational potential, it would also manifest itself as a dependence of the frequency difference of two clocks of different composition on gravitational potential,

$$
\Delta v / v=\left(\beta_{2}-\beta_{1}\right) \Delta U / \mathrm{c}^{2},
$$

where $v$ is the frequency difference between the two clocks, $U$ is the gravitational potential, and $\beta_{i}$ characterizes the anomalous redshift for clock $i$ ( $\beta_{\mathrm{i}}=0$ according to LPI).

A convenient source of a varying gravitational potential is that due to the earth's elliptical orbit about the sun, which results in a solar gravitational potential at the earth that varies over the course of a year sinusoidally with a peak-to-peak amplitude of $3.3 \times 10^{-10}$. Searches for this effect, then, can be carried out by looking for an annual oscillation in the relative frequency of clocks of two different species.

The best results to date for LPI tests using various pairs of atomic clocks are shown in Fig. 5 and Table I. The last two measurement are results we have obtained with our rubidium fountains after 1.2 years of operation. The high precision we obtain after a relatively short amount of time demonstrates the value of continuous operation for this type of measurement.

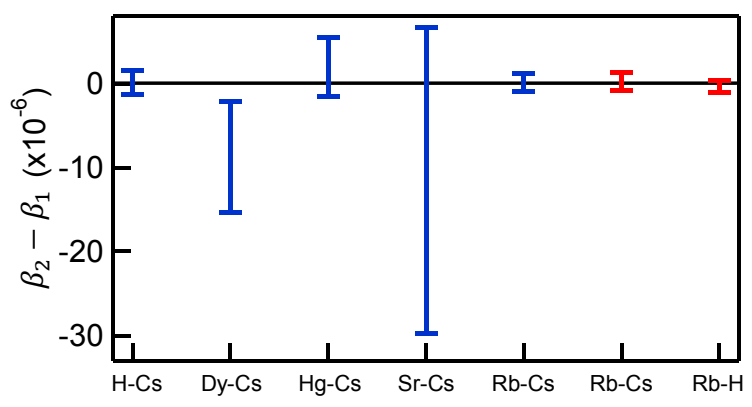

Figure 5. Summary of LPI tests using different pairs of atomic species. The last two measurements are those reported in this work. The data for this plot are shown in Table I.

TABLE I. SUMMARY OF LPI TESTS USING DIFFERENT PAIRS OF ATOMIC SPECIES.

\begin{tabular}{|c|cccc|} 
ref & species & years & $\left|\beta_{2}-\beta_{1}\right|\left(\times 10^{-6}\right)$ & uncert $\left(\times 10^{-6}\right)$ \\
\hline$[3]$ & H vs Cs & 7 & 0.1 & 1.4 \\
{$[4]$} & Dy vs Cs & 0.7 & 8.7 & 6.6 \\
{$[5]$} & Hg vs Cs & 5 & 2 & 3.5 \\
{$[6]$} & Sr vs Cs & 2.5 & 11.5 & 18.2 \\
{$[7]$} & Rb vs Cs & 14 & 0.1 & 1.0 \\
$*$ & Rb vs Cs & 1.2 & 0.3 & 1.1 \\
$*$ & Rb vs H & 1.2 & 0.4 & 0.7
\end{tabular}

Included in the table are the reference for each measurement (col 1); the pair of atomic species ( $\mathrm{col} 2$ ); the number of years used to make the measurement ( $\mathrm{col} 3$ ); and the values ( $\mathrm{col} 4)$ and uncertainties ( $\mathrm{col} 5)$ obtained. * This work.

\section{A. Rubdium vs Cesium}

We measure $\beta_{\mathrm{Rb}}-\beta_{\mathrm{Cs}}$ by comparing each of our unrecharacterized fountains, NRF4 and NRF5, to two different ensembles of cesium clocks, the local USNO cesium mean and the primary standards that contribute to TAI. Figure 6(a) shows a plot of the relative frequency of NRF4 versus the local cesium mean, with the best least-squares fit for an LPI signal included; the cesium mean has a small drift of about $2 \times 10^{-17} /$ day, which has been removed from the plot. The amplitude and statistical uncertainty for this fit are $-1.1 \pm 3.0 \times 10^{-16}$. Including all of the independent measurements, a final value for $\beta_{\mathrm{Rb}}-\beta_{\mathrm{Cs}}$ of $0.3 \pm 1.1 \times 10^{-6}$ is obtained.

\section{B. Rubidium vs Hydrogen}

We have made a preliminary LPI test using rubidium and hydrogen using one of the best masers available to us. This particular maser exhibited a drift of about $2 \times 10^{-17} /$ day over the past 14 months, which can be removed with little effect on the uncertainty of the resulting LPI fit. The relative frequency of 
NRF4 and this maser are shown in Fig. 6(b), along with the best-fit LPI signal. Combining this with a measurement using NRF5 gives a preliminary value of $\beta_{\mathrm{Rb}}-\beta_{\mathrm{H}}$ of $-3.5 \pm 7.1 \times 10^{-7}$. This particular maser is also recorded with a lower-noise measurement system, which should result in a more stringent limit than this preliminary value.
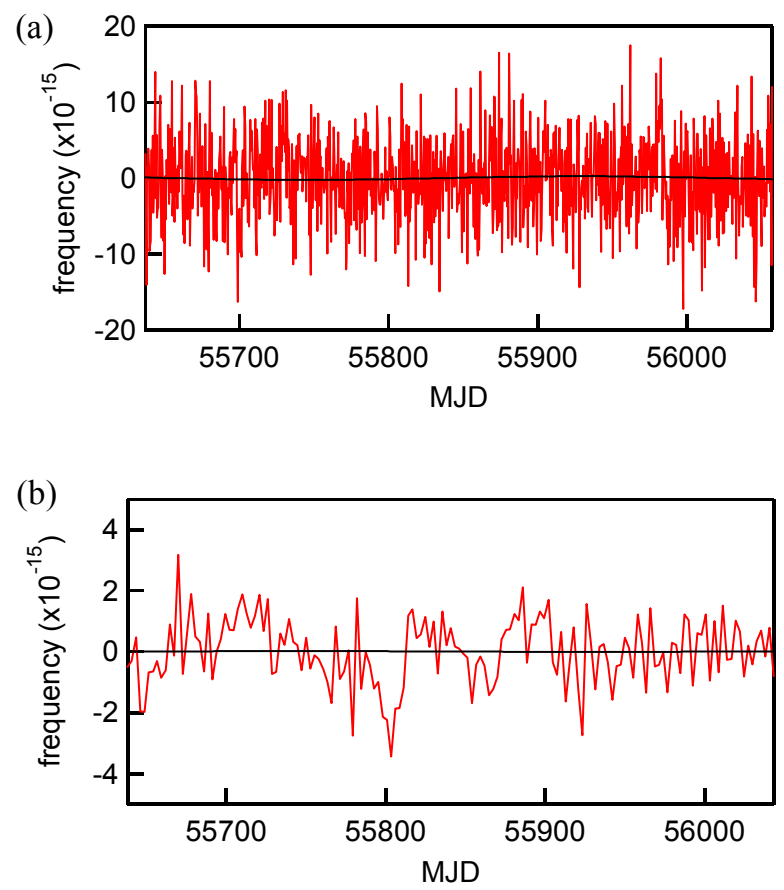

Figure 6. Plots of relative frequency difference for LPI tests using rubidium versus (a) cesium and (b) hydrogen. The black lines are leastsquares fit results for an annual oscillation with fixed frequency and phase.

Most masers drift much more significantly than the one used for this measurement, and the process of fitting in the presence of that drift, or removing the drift and fitting to the residuals, introduces an additional uncertainty that we are still working to definitively quantify. Most likely, the added uncertainty will be large compared to the low-drift masers, resulting in the final weighted average to be dominated by the lower-drift clocks. We also are considering whether it is best to use a maser mean rather than several measurements against independent masers, and the effects of flicker and random walk on the final uncertainty are also still being considered.

\section{CONCLUSIONS}

Four rubidium fountains at USNO have been in operation for more than a year and are contributing to UTC(USNO) and are being reported to the BIPM. A timescale generated from the four fountains indicates a stable frequency with respect to the primary standards that contribute to TAI. Tests of Local Position Invariance are facilitated with continously running clocks, as demonstrated with measurements of rubidium versus cesium and rubidium versus hydrogen over the past 14 months.

\section{ACKNOWLEDGEMENTS}

We are grateful for assistance from and discussions with Paul Koppang and Jim Skinner. Funding for the fountain project is from SPAWAR.

\section{REFERENCES}

[1] C. Ekstrom, S. Peil, T. Swanson, S. Crane, in Frequency Standards and Metrology: Proceedings of the Seventh Symposium, Lute Maleki, ed., World Scientific, New Jersey, pp. 308-313 (2008).

[2] Felicitas Arias, private communication; G. Panfilo, A. Harmegnies, L. Tisserand, Metrologia 49, 49-56 (2012).

[3] N. Ashby et al., Phys. Rev. Lett. 98, 070802 (2007).

[4] S. J. Ferrell, et al., Phys. Rev. A 76, 062104 (2007).

[5] T. M. Fortier, et al., Phys. Rev. Lett. 98, 070801 (2007).

[6] S. Blatt, et al., Phys. Rev. Lett. 100, 140801 (2008).

[7] J. Guena, et al., arXiv:1205.4235v1 (2012). 